\title{
General Practice Observed
}

\section{Management of backache in general practice}

\author{
A E DOSSETOR
}

British Medical fournal, 1975, 4, 32-33

The type of backache I see most is the result of a forward strain from either lifting, pulling, or falling doubled up. As I am particularly interested in the care of back injuries I may see as many as seven or eight some days, but average about 15 a week, from a group practice of 13500 patients at risk.

The treatment consists of (a) manipulation, followed by $(b)$ postural advice during the healing process, and (c) advice on avoiding a recurrence.

Some years ago I tried to get patients upright from the bentover back pain position, as illustrated in the lumbago advertisements, by suggesting they swung on a cold water pipe over my surgery door, while I wrote them out a prescription. The patients who became dramatically better "felt a click" in their backs; the others did not. I subsequently found that the tender posterior vertebral spine was less prominent after the "click". Hence I devised the following method of manipulation described here. One successful manipulation is often sufficient to cure the backache, whereas in Doran and Newell's study ${ }^{1}$ an average of six manipulations was required.

\section{Traction}

The patient is hung from a horizontal bar $2.1 \mathrm{~m}$ from the floor and above the doorway lintel. Hanging by arms allows the weight of the body below the lesion to widen the space between the vertebrae and remove the pressure from the segmental nerve. This in turn relieves the pain and the muscle spasm relaxes.

In heavy people with weak musculature this "hanging traction" often also realigns the vertebrae, and they are cured without manipulation. These patients are usually obese women. Conversely, light muscular men tend not to lose their muscle spasm as the traction of the weight of their lower half is not sufficient to separate their vertebrae, and most of those in whom pain is not alleviated are in this group. They are usually treated with physiotherapy.

Some patients with minor lesions need no more treatment, and $I$ advise patients to climb up and hang from the top of a door and wriggle their backs whenever their backs "go." Often they will manage to get upright and be relatively painfree while healing is taking place.

\section{Manipulation}

The tender posterior vertebral spine must be located and pushed forwards over the one below. The patient is swung to

The Rookery Medical Centre, Newmarket, Suffolk CB8 8NW

A E DOSSETOR, MB MRCGP, general practitioner

and fro while hanging from the bar (if necessary with his knees bent and his feet pointing to the ground), which will further release any muscle spasm and make the back pliable and more amenable to manipulation. Then with the left arm round the thighs the right fist is placed over the tender vertebral spine and a firm forward shove is applied with the right fist while the left arm pulls back on the thighs. Pressure is applied up to about $23 \mathrm{~kg}$ or till a movement is felt in the back. Patients describe this movement as "a click," which is very descriptive of what both patient and manipulator feel. Over-manipulation is prevented by the restraining influence of the undamaged vertebral ligaments and indeed the articulation between the inferior articular processes and the corresponding superior articular facets.

In the elderly only about $4.5 \mathrm{~kg}$ pressure should be applied, but the mechanics of forward back sprain apply in a back that has had some wear just as it does in the back of a young man. (Pressures have been measured on a spring.) Most manipulations are painless but about a quarter of the patients feel a sharp twinge; this pain goes after two or three hours, any residual pain being due to the manipulation not succeeding. Unsuccessful manipulation results from too gentle manipulation rather than too forceful, and if one is not successful the first time, muscle spasm (resulting from any twinge of pain produced) makes a second manipulation more difficult.

$X$-ray films show that Schmorl's nodes seem to be one reason for unstable intervertebral joints, making a backward displacement of one vertebra over the one below more likely.

Ideally $x$-ray films should be taken and the erythrocyte sedimentation rate estimated. The latter could be done by the surgery nurse and if abnormal, prudence dictates that an $x$-ray picture of the appropriate part should be taken before manipulation.

(A sprained spondylitis should be manipulated only gently. The traction effect of swinging from a bar is, however, harmless, and it alone will often allow the patient to get upright and then, with good posture, recover.)

\section{Advice to ensure healing}

Patients are advised to keep their spine in a neutral position, bent neither forwards, backwards, nor sideways. This upright position is maintained without effort while walking or standing, but chairs or beds must be specially shaped. Patients whose thoracic spines are sprained need to sit upright and have their mattresses supported on boards, especially at the level of injury, but those who have sprained their lumbar region need a convex support. Patients with a lumbar lesion are told to sit back in a chair with a firm seat and arms to help get up, put a cushion in the hollow of their backs, and sit back over the cushion. In bed they must sleep across a pillow "the size of their seats"- that is, patients with big buttocks and pronounced lumbar curve need a bigger pillow than patients with small buttocks and a shallower lumbar curve. When they wake in the morning they must not sit up but pull their knees up and roll out of bed, 
keeping their backs posturally upright. They need help with tights, but shoes, socks, and stockings may be put on by pulling the foot backwards. Hot baths help as the patients will float out into a good posture and the heat will soothe, but to get out of the bath they must turn over and kneel in the bath and not pull themselves out forwards.

To avoid a recurrence patients are told to take a forward strain on their backs by bending their knees and hips but not their backs before lifting, and if they cannot reach the object to be lifted they must get on one knee and still keep their backs posturally upright. They must also treat all objects to be lifted as if they were heavy - that is, they must not lift a weight carelessly off the floor but face the weight and lift as instructed.

Patients visited at home and found immobile in bed usually have a lumbar strain. A pillow is put under the lumbar region. While they hold the head of the bed traction is applied to their legs until their muscle spasm relaxes and the pain goes. They are then rolled out of bed and shown how they can manage if they stay upright. Many of these patients, lying across a pillow, will turn and manipulate their own backs, usually at night, but the more courageous are hung on a door and manipulated.

This treatment could have been deduced by realising how strong a leverage action is exerted to slip back one vertebra over the one below if the muscles do not hold during a forward strain. This is not easily noted, because $x$-ray films of the vertebral column are notoriously unreliable and such movements that the vertebrae sustain, although enough to irritate the spinal nerve, are readily passed as "within normal limits."

Having manipulated well over 2000 backs in this way (patients with unstable backs come back for each relapse) and as only a few are off work for more than a week (some go straight back to work), I would like to recommend this technique for the treatment of backache.

\section{Reference}

' Doran, D M L, and Newell, D J, British Medical Fournal, 1975, 2, 161

\title{
Today's Treatment
}

\section{Diseases of the central nervous system}

\author{
Pharmacological basis of treatment
}

\author{
JOHN C GILBERT
}

British Medical fournal, 1975, 4, 33-35

In introducing and summarising a subject as complex as the pharmacological basis of treatment of disorders of the nervous system brief reference has to be made to the general direction of progress, the problems, and the discoveries which gave the subject impetus.

Progress in treating some disorders has been long delayed by inadequate experimental methods and by the lack of suitable animal models of human disease. Experiments involving electrical stimulation of the dental pulp, or the radiant heat tailflick test and mouse hot-plate test of nociception in animals, have proved to be useful models for testing potential analgesics for man, but some types of pain do not appear to have equivalent sensory stimuli in animals and different types of pain require different drugs for their management in man. Measurement of seizure thresholds, inducing the seizure activity by leptazol or electroshock, has its value in determining the anticonvulsant activities of drugs but the features of the seizures are not identical to those of an epileptic attack. No animal model can be claimed to be representative of schizophrenia in man. Given this sort of impediment, the development of therapeutic drugs has been a checkered affair and some drugs are in current use not so much as a result of logical study as of empirical observa-

tion. For example, the narcotic analgesic pethidine aroused interest originally because of its atropine-like effects and its tossible value as an antispasmodic. It was only later that the effects of pethidine on the central nervous system (CNS), resulting in sedation, analgesia, and euphoria (features commonly associated with morphine administration), were noted, suggesting its possible therapeutic use as an analgesic.

While the mechanisms of action of many drugs used in treating diseases of the nervous system are not clear, some modify quite distinct chemical transmission processes and the metabolic pathways sustaining these processes have been identified.

\section{Myasthenia gravis}

The metabolic pathways involving acetylcholine-its synthesis through choline acetyltransferase and its destruction through acetylcholinesterase-are well established, and the treatment of myasthenia gravis relies heavily on inhibitors of acetylcholinesterase because of the role of acetylcholine as a transmitter at the neuromuscular junction. Myasthenic patients tend to show an increased response to neuromuscular blocking drugs which competitively antagonise the action of acetylcholine at the inotor end-plate and a decreased response to depolarising blocking agents such as suxamethonium.

The symptoms of myasthenia are compatible with diminished activity at the motor end-plate, and evidence suggests that this results more from a decrease in the amount of transmitter released after presynaptic events than from abnormal (reduced) sensitivity of the end-plate nicotinic receptor region to the transmitter impinging upon it. In either case prevention of destruction of acetylcholine by inhibition of the postsynaptically sited esterase 